\title{
Sorting out the roles of PPAR $\alpha$ in energy metabolism and vascular homeostasis
}

\author{
Philippe Lefebvre, Giulia Chinetti, Jean-Charles Fruchart, and Bart Staels
}

Département d’Athérosclérose, Institut Pasteur de Lille, INSERM U545, and Université de Lille 2, Lille, France.

\begin{abstract}
PPAR $\alpha$ is a nuclear receptor that regulates liver and skeletal muscle lipid metabolism as well as glucose homeostasis. Acting as a molecular sensor of endogenous fatty acids (FAs) and their derivatives, this ligand-activated transcription factor regulates the expression of genes encoding enzymes and transport proteins controlling lipid homeostasis, thereby stimulating FA oxidation and improving lipoprotein metabolism. PPAR $\alpha$ also exerts pleiotropic antiinflammatory and antiproliferative effects and prevents the proatherogenic effects of cholesterol accumulation in macrophages by stimulating cholesterol efflux. Cellular and animal models of PPAR $\alpha$ help explain the clinical actions of fibrates, synthetic PPAR $\alpha$ agonists used to treat dyslipidemia and reduce cardiovascular disease and its complications in patients with the metabolic syndrome. Although these preclinical studies cannot predict all of the effects of PPAR $\alpha$ in humans, recent findings have revealed potential adverse effects of PPAR $\alpha$ action, underlining the need for further study. This Review will focus on the mechanisms of action of PPAR $\alpha$ in metabolic diseases and their associated vascular pathologies.
\end{abstract}

\section{Introduction}

Nutrient metabolism and energy homeostasis are tightly regulated by endocrine, paracrine, and autocrine signals that control the expression and activity of key metabolic enzymes and transport proteins by transcriptional and posttranscriptional mechanisms. Lipid mediators play a critical role in metabolic control, and the PPARs (NR1Cs), a class of ligand-activated transcription factors, have emerged as master transcriptional regulators of lipid and carbohydrate metabolism. Saturated and unsaturated long-chain fatty acids (FAs) and their eicosanoid derivatives are natural activators of this subclass of nuclear receptors. Increased recognition of a role for PPARs in metabolic regulation came following the discovery that the hypolipidemic fibrates and the insulin sensitizers thiazolidinediones were synthetic ligands for PPAR $\alpha$ (NR1C1; refs. $1,2)$ and PPAR $\gamma$ (NR1C3; ref. 3), respectively. PPAR (NR1C3), also known as PPAR $\beta$, is the third PPAR isotype.

Accumulating evidence supports a link between the 3 PPARs and diabetes, obesity, dyslipidemia, and inflammation. PPAR $\alpha$ controls liver and skeletal muscle lipid metabolism, and glucose homeostasis. PPAR $\alpha$ influences intracellular lipid and carbohydrate metabolism through direct transcriptional control of genes involved in peroxisomal and mitochondrial $\beta$-oxidation pathways, FA uptake, and triglyceride (TG) catabolism. Moreover, preclinical data suggest a role for PPAR $\alpha$ in body weight control, supporting the use of PPAR $\alpha$ agonists to treat obesity (4). Mice deficient in PPAR $\alpha$ exhibit a delayed response to inflammatory stimuli (5). Several clinical trials demonstrate the efficiency of fibrates at decreasing circulatory inflammatory markers and reducing the progression of coronary atherosclerotic lesions. The ability of PPAR $\alpha$ to improve

Nonstandard abbreviations used: $\mathrm{ABCA} 1, \mathrm{ABC}$ transporter $\mathrm{A} 1$; AF, activating function; CE, cholesteryl ester; CPT-1, carnitine palmitoyl transferase 1; CRP, C-reactive protein; FA, fatty acid; FAO, FA oxidation; FIELD, Fenofibrate Intervention and Event Lowering in Diabetes; LBD, ligand-binding domain; LPL, lipoprotein lipase; MCP-1, monocyte chemoattractant protein-1; NR, nuclear receptor; PPRE, PPAR response element; RCT, reverse cholesterol transport; RXR, retinoid X receptor; sdLDL, small dense LDL; SR, scavenger receptor; TG, triglyceride.

Conflict of interest: The authors have declared that no conflict of interest exists. Citation for this article: J. Clin. Invest. 116:571-580 (2006). doi:10.1172/JCI27989. symptoms of the metabolic syndrome (visceral obesity, insulin resistance, atherogenic dyslipidemia, and inflammation) suggests that PPAR $\alpha$ may be beneficial in the prevention or treatment of type 2 diabetes mellitus and associated complications.

\section{Structure of PPAR $\alpha$}

PPAR $\alpha$ has a functional domain structure analogous to those of other nuclear receptor (NR) superfamily members. Like steroid receptors, PPAR $\alpha$ interacts with hsp90 (6). The central DNAbinding domain (DBD) of PPAR $\alpha$ is flanked by an N-terminal region called activating function-1 (AF-1) (7) that is activated by phosphorylation, as shown by insulin-stimulated AF-1 phosphorylation (S1). The DBD confers to PPAR $\alpha$ the ability to bind to PPAR response elements (PPREs) in the promoter of target genes as an obligate heterodimer with retinoid $X$ receptor (RXR) isotypes (8). PPREs typically are organized as direct repeats of the core sequence AGGTCA separated by 1 or 2 nucleotides (DR1 and DR2), flanked upstream by A/T-rich sequences (S2). While PPRE geometry ensures specificity for PPAR/RXR heterodimers, DR1 and DR2 PPREs are also recognized by RXR homodimers or retinoic acid receptor (RAR)/RXR heterodimers, suggesting cross-talk with RARs and RXRs that may influence metabolic control (9). The C-terminus of PPAR $\alpha$, whose 3D structure has been solved $(10,11)$, contains the ligand-regulated $\mathrm{E}$ domain or AF-2 or ligand-binding domain (LBD), which harbors a large T-shaped ligand-binding pocket $\left(1,300 \AA^{3}\right)$ to accommodate various natural and synthetic ligands.

\section{Transcriptional activation by PPAR $\alpha$}

The transactivation process by NRs relies on 5 major steps: ligand binding; stable binding of liganded NR to DNA; corepressor dismissal and coactivator recruitment; activation of transcription; and dissociation of the transcriptional complex, followed by either shut-down or reinitiation of transcription. Crystallographic studies suggest that ligand binding to PPAR $\alpha$ induces a global stabilization of the receptor conformation (11), without major structural reorganization, unlike the prototypical retinoic acid receptor $\mathrm{LBD}$ that undergoes major structural transitions upon 


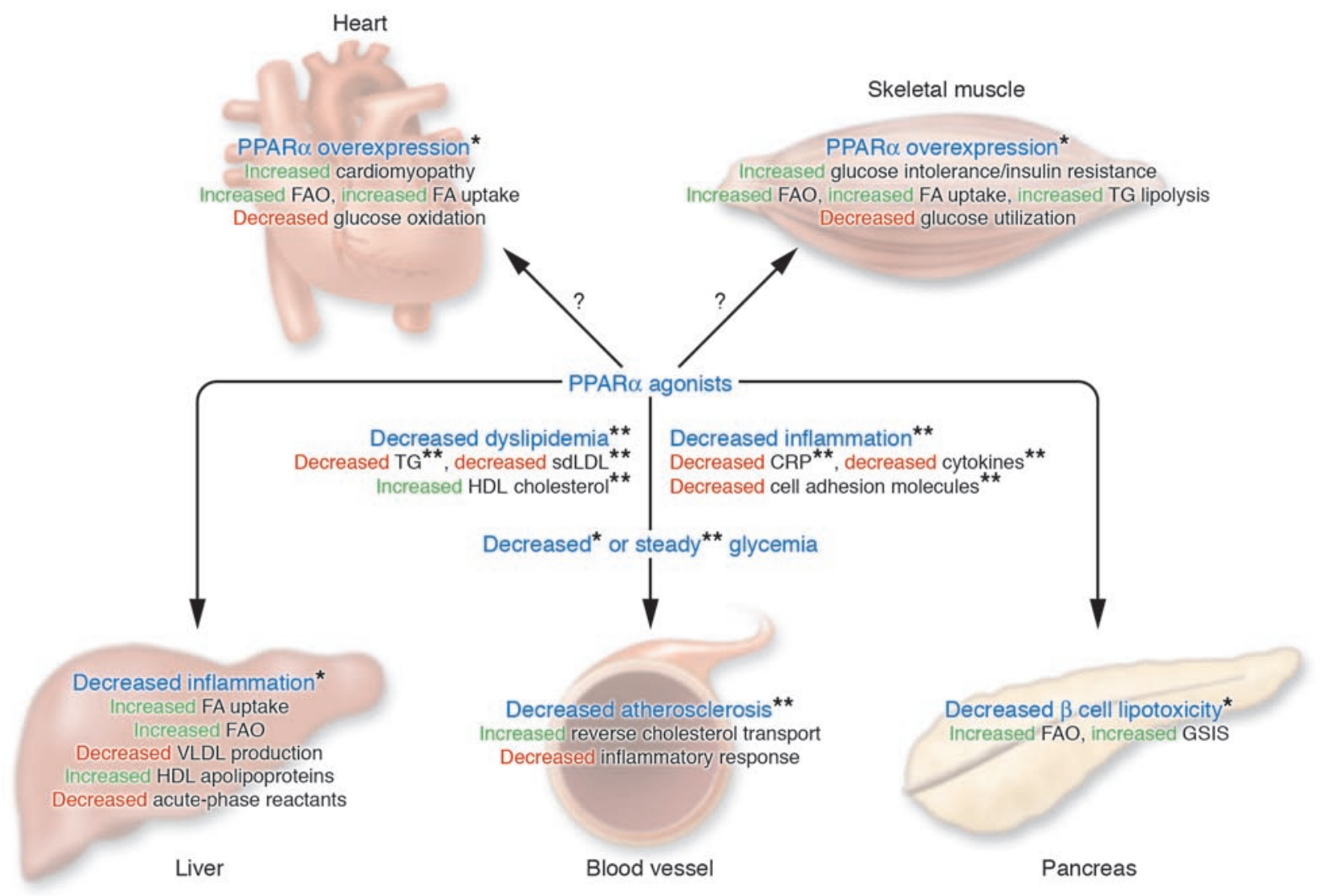

Figure 1

Metabolic actions of PPAR $\alpha$ and potential pathophysiological consequences. The main effects of PPAR overexpression or of PPAR $\alpha$ ligands in mice (denoted by a single asterisk) and in humans (denoted by a double asterisk) are shown. GSIS, glucose-stimulated insulin secretion.

agonist binding (S3). This suggests that the major contribution of the PPAR $\alpha$ ligand is the stabilization of a predefined structure able to engage protein-protein interactions with coactivators (agonistbound PPAR $\alpha$ ) or corepressors (antagonist-bound PPAR $\alpha$ ) (11). This feature is common to PPAR $\alpha, \operatorname{PPAR} \delta$, and PPAR $\gamma$ (S4, S5), but a recent refinement of the structure of the LBD of PPAR $\delta$ showed that this polypeptide is able to trap endogenous bacterial FAs prior to crystallization (S6). Therefore, the possibility arises that PPAR $\alpha$, and other isotypes, might shift from an inactive to an active conformation, similarly to other NRs. However, it is not known whether PPAR $\alpha$ is, in a biological context, constitutively bound to endogenous FAs.

Coactivator and corepressor complexes possess distinct enzymatic activities (such as acetylase, deacetylase, methylase, demethylase, and kinase activities) targeting chromatin, components of the basal transcriptional machinery, and other coactivators and corepressors. The orchestrated recruitment and dismissal of coactivators and corepressors leads to chromatin decompaction and preinitiation complex assembly on promoters. The transcriptional response is also strongly influenced by the chemical structure of the ligand, the nature of the PPRE (12), the structure of the promoter, and the expression of coactivators and corepressors in a given cell type. The direct interaction of coactivators and corepressors with PPAR $\alpha$ requires 1 or more cores of a degenerated LXXLL motif on the coregulator protein, and several proteins have features of a bona fide coactivator or corepressor for PPAR $\alpha$ (S7-S13). However, none has been proven essential for PPAR $\alpha$-induced transcription, including the prototypical SRC1 molecule (13), reflecting a likely functional redundancy between coactivators, or the lack of appropriate models to study such mechanisms. This general mechanism for transcriptional activation by PPAR $\alpha$ is likely similar for other PPAR $\alpha$ target genes.

\section{Gene repression by PPAR $\alpha$}

PPAR $\alpha$ also interferes negatively with other nuclear signaling pathways such as the AP1 (14) and NF-кB pathways. Indeed, PPAR $\alpha$ inhibits genes induced by NF- $\mathrm{B}$, such as VCAM-1, COX-2, and IL-6 $(15,16)$, providing a molecular basis for the antiinflammatory effects of PPAR $\alpha$ ligands in vivo. PPAR $\alpha$ upregulates expres-

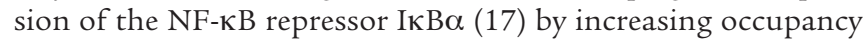

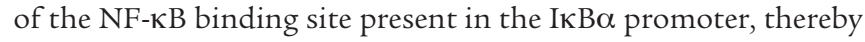
potentiating a negative feedback loop. This occurs independently of PPAR $\alpha$ binding to DNA and thus could involve direct proteinprotein interaction of PPAR $\alpha$ with the NF- $\kappa B$ complex (18). A similar mechanism has been described for the fibrate-mediated inhibition of IL-1-induced expression of C-reactive protein (CRP) (19). Interference of PPAR $\alpha$ with the CAATT/enhancer binding protein $(\mathrm{C} / \mathrm{EBP})$ signaling pathway is the molecular basis for the inhibition of IL- 6 -induced fibrinogen- $\alpha$ and $-\beta$ and of serum amyloid A expression (20). PPAR $\alpha$ also decreases the expression of IL-6 receptor components as well as that of C/EBPs (21). 
The biological and therapeutic activities of PPAR $\alpha$ are therefore the result of the combination of both transactivating and transrepressive properties of this receptor. In addition, posttranslational modifications are important regulatory controls. SUMOylation and acetylation regulate transrepressive and transactivating activities of some NRs, and phosphorylation may inhibit transrepression by PPAR $\alpha$. PKC inhibition increases repression of the fibrinogen- $\beta$ gene by PPAR $\alpha$ by modulating the phosphorylation state of the PPAR $\alpha$ D domain (22). The ability of NRs to regulate transcription is also a function of promoter architecture, ligand structure, cell type, and physiological and pathological conditions. This raises the possibility of designing ligands with dissociated transactivating and transrepressive activities, enabling specific targeting of gene subsets. However, despite extensive knowledge of PPAR $\alpha$ molecular biology, the design of such ligands remains purely empirical.

\section{Mechanisms controlling PPAR $\alpha$ activity}

There are several levels at which PPAR $\alpha$ activity can be controlled. These include the regulation of its expression, the nature of the ligand, the levels of coactivators and corepressors, and posttranslational modifications of PPAR $\alpha$ and the associated coactivators and corepressors. Temporal expression of PPAR $\alpha$ in rats is controlled by the circadian clock (23), through the positive control of PPAR $\alpha$ expression by glucocorticoids $(24,25)$ and the clock gene Bmal1 (26). PPAR $\alpha$ expression is induced during fasting in Sv129 mice $(27,28)$, and influenced by hormonal signals such as leptin, growth hormone, and insulin $(24,29,30)$. Synthetic PPAR $\alpha$ ligands such as Wy14,643, GW7647, or fibrates increase the half-life of the PPAR $\alpha$ polypeptide by preventing its ubiquitination and its subsequent degradation via the proteasome (31).

Human PPAR $\alpha$ promoter activity is induced by PPAR $\alpha$ itself and by the nuclear receptor HNF4, a major regulator of gluconeogenesis (32). Glucose decreases PPAR $\alpha$ expression in the pancreas, leading to diminished fatty acid oxidation (FAO) and TG accumulation, a supposed cause for pancreatic lipotoxicity (33).

Extracellularly regulated signaling pathways impact on PPAR $\alpha$ through phosphorylation. The role of PKC in specifying PPAR $\alpha$ transcriptional activities has been described above, and a phosphorylation-dependent increase in PPAR $\alpha$ activity by the stressactivated p38 protein kinase has been shown to favor PPAR $\alpha$ mediated transactivation (34).

\section{Endogenous PPAR $\alpha$ ligands}

The quest for "the" endogenous PPAR $\alpha$ ligand is still ongoing. Early reports identified mono- and polyunsaturated FA as well as eicosanoids as natural PPAR $\alpha$ ligands $(2,35)$. Long-chain fatty acyl-CoAs and saturated FAs also bind and activate PPAR $\alpha$ with $\mathrm{EC}_{50}$ values in the high-micromolar range $(36,37)$. Recently, Chakravarthy and colleagues reported that liver-specific inactivation of the fatty acid synthase (FAS) gene in mice resulted in a phenotype identical to that of fasting PPAR $\alpha$-deficient mice (38). Defects in FAO, cholesterol metabolism, and gluconeogenesis could be corrected by PPAR $\alpha$ agonist treatment in FAS-deficient mice, suggesting that de novo synthesized end products of FAS, including palmitate, regulate PPAR $\alpha$ activity. The data also suggest that FAs released from adipocytes are inactive with respect to PPAR $\alpha$ and thus exert physiologically distinct activities.

\section{PPAR $\alpha$ and intracellular lipid metabolism}

PPAR $\alpha$ is highly expressed in tissues displaying a high catabolic rate of FAs, such as the liver, skeletal muscles (mostly in slowtwitch, oxidative type I fibers [S14]), brown fat, heart, kidneys, and cells of atherosclerotic lesions (endothelial cells, smooth muscle cells, monocytes/macrophages). In rodents, PPAR $\alpha$ activation leads to peroxisome proliferation and hepatocarcinoma, a property intrinsic to mouse PPAR $\alpha$ and, fortunately, not observed in humans (39). Targeted disruption of the PPAR $\alpha$ gene in mice revealed its role in mitochondrial and peroxisomal FA $\beta$-oxidation (FAO), FA uptake, and lipoprotein assembly and transport $(27,28,40-46)$. While the phenotype of PPAR $\alpha$-deficient mice fed ad libitum is mild, fasting or inhibition of mitochondrial FA import severely impairs FA uptake and FAO, leading to sex-specific liver steatosis and cardiac lipid accumulation, hypoglycemia, and hypothermia $(28,43)$.

PPAR $\alpha$ in hepatic lipid metabolism. Short-term adjustment of mitochondrial FA $\beta$-oxidation occurs through regulation of carnitine palmitoyl transferase 1 (CPT-1), which controls FA import into the mitochondria. CPT- 1 expression is regulated by PPAR $\alpha$ in liver and myocytes (44), as well as that of major enzymes of the $\beta$-oxidation pathway (acyl-CoA synthetase, very-long- and medium-chain acyl-CoA dehydrogenases, 3-ketoacyl-CoA thiolase). Partial oxidation of very-long-chain and long-chain FAs, as well as of other lipid derivatives such as eicosanoids or branched FAs, occurs in peroxisomes to provide substrates for mitochondrial oxidation. The expression of key enzymes catalyzing the degradation of straight-chain FAs (acyl-CoA oxidase, L-bifunctional protein, thiolase) in peroxisomes is regulated by PPAR $\alpha$. The mitochondrial HMG-CoA synthase, which converts acetylCoA units into ketone bodies during fasting or diabetes, is also upregulated by PPAR $\alpha$ (45). Thus PPAR $\alpha$ acts in liver and other organs to reduce intracellular FA concentrations, likely contributing to decreased VLDL particle production and plasma TG levels in patients treated with an agonist. PPAR $\alpha$ 's role in energy homeostasis is thus clearly demonstrated in animal models, but unclear at present in humans. Moreover, the lower expression of PPAR $\alpha$ in human compared with rodent liver (47), as well the dominant-negative splice variant of PPAR $\alpha$ in human liver (48), suggests a more modest role of PPAR $\alpha$ in humans.

PPAR $\alpha$ and cellular FA uptake. PPAR $\alpha$ also modulates FA cellular uptake. Fatty acid translocase, or CD36, is a glycoprotein regulating FA uptake in multiple cell types, including hepatocytes, adipocytes, and monocytes, as well as cells in muscle and intestine. PPAR $\alpha$ activation upregulates CD36 expression in liver and intestine, but not in skeletal muscle (49). Similarly, expression of the fatty acid transport protein, an integral membrane protein involved in FA uptake, is upregulated by PPAR $\alpha$ activation in hepatocytes (46).

PPAR $\alpha$ in cardiac lipid metabolism. The hearts of PPAR $\alpha$-deficient mice express very low levels of mitochondrial FAO enzymes, relying almost exclusively on glucose oxidation for energy, similarly to fetal hearts $(42,50,51)$. A PPAR $\alpha$-dependent transcriptional network is activated in the heart during the transition from fetus to newborn, creating a metabolic switch from glucose to FAO (S15). Moreover, the metabolic rate of FA is increased in wild-type cardiomyocytes upon treatment with a synthetic PPAR $\alpha$ agonist (52), and cardiac overexpression of PPAR $\alpha$ leads to upregulation of FAO enzymes, and to downregulation of enzymes controlling glucose uptake and oxidation (51). The cardiac hypertrophy and dysfunc- 
tion in these PPAR $\alpha$-overexpressing mice thus resemble the cardiac phenotype of diabetic mice, and cardiac as well as age-related liver insulin resistance is observed in these mice (53). Thus PPAR $\alpha$ plays a regulatory role in controlling cardiac metabolic switches. A decreased expression of PPAR $\alpha$ in the nondiabetic, hypertrophic heart alters FAO in cardiomyocytes and may contribute to cardiac dysfunction (54), whereas a decreased PPAR $\alpha$ expression in the diabetic heart may be a mechanism to protect the heart from further oxidative stress-induced damage due to excessive FAO (55).

Extrapolating these findings in mice to human pathology is difficult. The NR field has provided ample evidence that ligand-mediated activation of a receptor can trigger biological responses distinct from those that result from receptor overexpression (see ref. 51, for example). Similarly, the outcomes of gene inactivation studies are not always predictable. Moreover, the metabolic response (such as TG lowering) to agonist-induced systemic PPAR $\alpha$ activation may alter cardiac metabolism. Nevertheless, data in genetic models suggest that chronic activation of PPAR $\alpha$ can lead to ventricular dysfunction, and recent evidence shows that treating cardiomyopathic mice with fenofibrate worsened heart function (56). However, an important unresolved issue is the contribution of peroxisome proliferation to the cardiac phenotype, which occurs in mice but not humans (39). Thus, although there is no evidence that such events occur in humans treated with weak PPAR $\alpha$ agonists (i.e., fibrates), monitoring of cardiac function in diabetic patients appears to be indicated for potent PPAR $\alpha$ agonists.

\section{Lipoprotein metabolism}

$P P A R \alpha$ and TG and LDL metabolism. The therapeutic benefit of fibrates is due in part to reduced VLDL production and enhanced catabolism of TG-rich particles, which indirectly decreases small dense LDL (sdLDL) particles, enhancing the formation of HDL particles and hepatic elimination of excess cholesterol. Fibrates have a marked effect on VLDL and chylomicron TG hydrolysis mediated by PPAR $\alpha$, which upregulates lipoprotein lipase (LPL) transcription in liver and muscle (57). LPL is a triacylglycerol hydrolase in the capillary endothelium of peripheral tissues, where its inactivation leads to severe hypertriglyceridemia and decreased HDL formation (S16). Another control of TG catabolism is regulation of the ratio of lipolytic versus antilipolytic apolipoprotein content in TG-rich particles. ApoC-III is an inhibitor of both LPL activity and remnant clearance, and apoC-IIIoverexpressing mice are severely hypertriglyceridemic (S17). ApoC-III synthesis is lowered by PPAR $\alpha$ agonists in murine and human hepatocytes, both in vivo and in vitro $(42,58)$, thereby favoring VLDL lipolysis and generation of large LDL particles that are more efficiently cleared via the LDL receptor. Interestingly, expression of the recently identified apoA-V, a potent activator of lipolysis, is upregulated by PPAR $\alpha$ agonists (59).

$P P A R \alpha$ and HDL metabolism. HDLs are protective against atherosclerotic vascular disease and are the main vehicle of reverse cholesterol transport (RCT). The interaction of HDL or apoA-I with scavenger receptor $\mathrm{BI}$ (SR-BI) and $\mathrm{ABC}$ transporter A1 (ABCA1), G1, or G4 triggers cholesterol efflux from peripheral tissues, and HDL particles direct cholesterol for hepatic excretion into the bile (60). Macrophage cholesterol efflux is of paramount importance for atherosclerosis, although it represents only a small fraction of the whole-body RCT. PPAR $\alpha$ agonists induce ABCA1 and SR-BI expression in macrophages $(61,62)$, thereby enhancing the first steps of macrophage RCT. Expression of the major human
HDL apolipoprotein genes apoA-I and apoA-II is activated in response to fibrate treatment in vitro $(63,64)$ and in humans $(65,66)$ via direct transcriptional control by PPAR $\alpha$. Devoid of any functional PPRE in its promoter, the murine apoA-I gene is negatively regulated by PPAR $\alpha$ agonists through an indirect pathway implicating the PPAR $\alpha$-dependent induction of the orphan NR Rev-erba, a negative regulator of transcription (67). This is an example of the major differences between rodent and human metabolic control by PPAR $\alpha$ (see below). Thus PPAR $\alpha$ activation, by virtue of its effects on the transcriptional activities of genes involved in lipoprotein metabolism, elicits a global normolipidemic response, by reducing TG-rich particle production, increasing their lipolysis, and promoting HDL metabolism and RCT. These collective effects should enhance transport of cholesterol from peripheral tissues to the liver.

\section{Glucose metabolism}

It is well established that excess fat intake promotes insulin resistance, resulting in increased gluconeogenesis and hepatic glucose production. In addition, hepatic and peripheral tissue lipotoxicity is a major causative factor for the development of type 2 diabetes mellitus. TGs provide the gluconeogenesis pathway with the essential substrate, glycerol, in addition to acetyl-CoA, reducing equivalents and ATP.

The mild PPAR $\alpha$-deficient phenotype becomes pronounced upon exposure to thermic, metabolic, or inflammatory stress (28, 43). The severe hypoglycemia observed specifically in PPAR $\alpha$-deficient mice upon fasting, characterized by a $50 \%$ drop in blood glucose concentration after 24 hours of fasting, suggested a role for PPAR $\alpha$ in glucose homeostasis (28). Several mechanisms may account for this fasting hypoglycemia, including normal glucose6-phosphate production in liver accompanied by the shift from glucose to glycogen production (68). Other authors attribute the fasting hypoglycemia to decreased production of lactate and hepatic glucose (69). Fasting induces the conversion of glycerol into glucose through the induction of several hepatic enzymes such as glycerol-3-phosphate dehydrogenase (GPDH) and glycerol kinase. The expression of these enzymes, and of the glycerol transporters aquaporins 3 and 9, is PPAR $\alpha$-dependent (70). GPDH deficiency in mice and humans leads to hypoglycemia, underlining the important role of glycerol as a substrate for glucose synthesis (S18, S19).

The mammalian ortholog of TRB3, another PPAR $\alpha$-target gene, is an important link between glucose and lipid metabolism. TRB3 is an inhibitor of Akt/protein kinase B, a positive regulator of cellular responses to insulin (S20). Upregulation of TRB3 expression through direct transcriptional control by PPAR $\alpha$ may impact negatively on liver insulin signaling, and in turn perturb glucose homeostasis (71). Moreover, glucocorticoid-induced diabetes is PPAR $\alpha$-dependent (72), and, accordingly, PPAR $\alpha$-deficient mice are protected from high-fat dietinduced insulin resistance $(73,74)$. Thus, PPAR $\alpha$ is a key player in hepatic glucose homeostasis.

The response to fasting is also dependent on the pancreas, and PPAR $\alpha$-deficient mice inefficiently suppress insulin secretion upon fasting, resulting in relative hyperinsulinemia (75). However, treatment of obese mice with PPAR $\alpha$ agonists improves insulin sensitivity and decreases blood glucose and insulin levels (76). A similar treatment of severely insulin-resistant lipoatrophic mice decreases blood glucose but does not normal- 


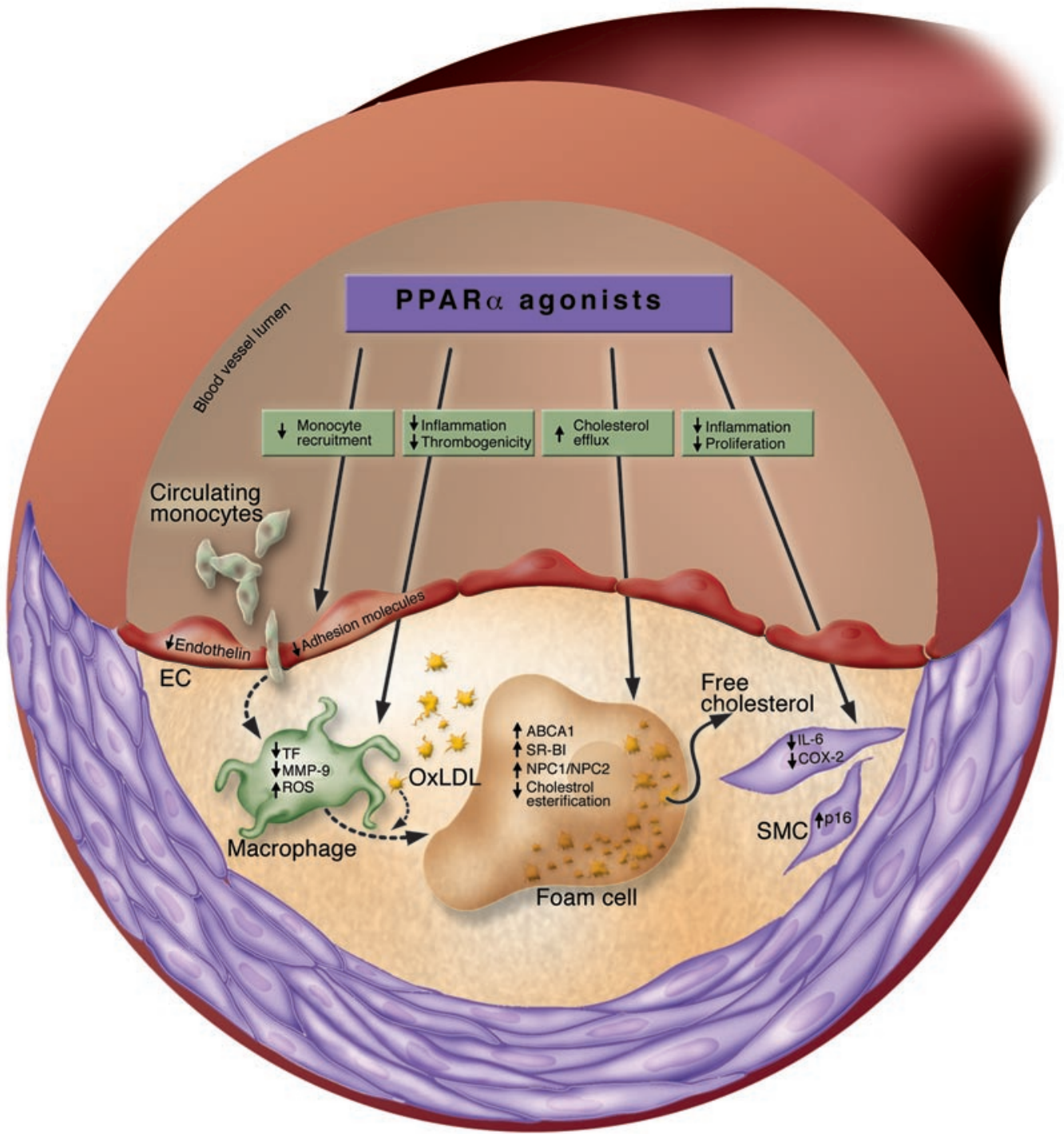

Figure 2

$\operatorname{PPAR} \alpha$ and atherosclerosis. The effects of PPAR $\alpha$ agonists in atherosclerosis are depicted for the most prominent cell type present in atherosclerotic lesions. NPC1 and 2, Niemann-Pick type $C$ proteins 1 and 2; OxLDL, oxidized LDL; SR-BI, scavenger receptor $\mathrm{BI}$; $\mathrm{TF}$, tissue factor.

ize insulin levels (77). In another model of lipoatrophy, PPAR $\alpha$ treatment improved both blood glucose and insulin levels (78). In prediabetic monkeys, lipoatrophic rats, or high-fat-fed rodents, PPAR $\alpha$ activation by a high-affinity agonist reversed insulin resistance, likely because of increased FA clearance from insulin-sensitive organs $(76,77,79,80)$. Interestingly, PPAR $\alpha$ activation in pancreatic islet $\beta$ cells also increases pancreatic FAO and potentiates glucose-induced insulin secretion, suggesting that PPAR $\alpha$ activation protects pancreatic islets from lipotoxicity (81). This raises the exciting yet untested hypothesis that PPAR $\alpha$ activation may prevent progression from a prediabetic, insulin-resistant state to type 2 diabetes.

While it is clear that PPAR $\alpha$ plays an obligatory role in liver and heart FAO, the importance of PPAR $\alpha$ in skeletal muscle FAO is obliterated by a compensatory role of the ubiquitous PPAR $\delta$ (82). However, overexpression of PPAR $\alpha$ in skeletal muscle in vivo impacts on peripheral glucose homeostasis. The increased FAO in PPAR $\alpha$-overexpressing muscles is accompanied by a decreased insulin-stimulated glucose uptake. Mice are resistant to diet-induced obesity but exhibit glucose intolerance, revealing a link between muscle PPAR $\alpha$-driven FAO and insulin resistance
(83). This suggests that PPAR $\alpha$ activation in skeletal muscles shifts substrate utilization from glucose to FA, a conclusion supported by loss-of-function experiments $(73,83)$. However, only a few clinical trials report an improvement of glucose homeostasis after fibrate treatment (84-87). Moreover, the recent Fenofibrate Intervention and Event Lowering in Diabetes (FIELD) study did not reveal any effect of fenofibrate on glucose parameters in diabetic patients (88); this suggests that effects on glucose homeostasis may be species specific. The multiple metabolic actions of PPAR $\alpha$ are summarized in Figure 1.

\section{PPAR $\alpha$ and atherosclerosis}

The identification of PPAR $\alpha$ expression in cell types of the atherosclerotic lesion has led to a thorough investigation of its modulatory role in this process. The gradual process of atherosclerotic lesion formation involves multiple cell types. Initiation occurs when large arteries are exposed to atherogenic stimuli, such as bacterial products, dyslipidemia, proinflammatory cytokines, or vasoconstrictor hormones such as angiotensin II. At this early stage, VCAM-1, activated through an NF-кB-dependent pathway, is believed to play a role in monocyte recruitment 
to nascent atherosclerotic lesions. Transmigration of monocytes and mast cells into the arterial intima, attracted by chemokines such as monocyte chemoattractant protein-1 (MCP-1) and IL-8, perturbs intercellular communications and promotes monocyte differentiation into macrophages and mast cell degranulation. Mast cells produce granule remnants rich in heparin proteoglycans, which interact with apoB-100, enhancing LDL retention. In this inflammatory milieu, SMCs migrate and proliferate, releasing MMPs that disrupt the ECM, exposing proteoglycans. Enhanced binding of lipoproteins to these proteoglycans favors their oxidation and glycation, perpetuating the inflammatory cycle. Macrophages, expressing scavenger receptors of the B class (CD36) and the A class (SR-A), internalize modified and oxidized LDL to form foam cells, which produce additional cytokines and growth factors. Following modified LDL uptake, cholesteryl esters (CEs) are continuously hydrolyzed by a CE hydrolase and re-esterified by acyl-CoA:cholesterol acyltransferase (ACAT). Eventually calcification occurs, particularly in patients on renal dialysis. In addition, apoptosis and necrosis of foam cells increase tissue factor, which can initiate thrombus formation. Formation of a necrotic core in the atherosclerotic plaque eventually progresses to plaque erosion and rupture, leading to clinical manifestations such as unstable angina, stroke, and myocardial infarction. However, when cholesterol acceptors are present in the extracellular fluid, RCT is initiated, and cholesterol flows out of cells, through the ABC transporters ABCA1 and $A B C G 1 / G 4$, which may reverse the atherosclerotic lesion.

Clinical trials as well as in vitro data provide compelling evidence that PPAR $\alpha$ acts as an antiatherogenic factor by interfering at multiple stages of the atherosclerosis process (Figure 2). Results from animal models have yielded conflicting results, which may be due to inherent differences in the models, and the species differences found in rodent and human metabolisms (74, 89-92). In the absence of inflammatory stimuli, PPAR $\alpha$ may promote proatherogenic responses. The expression level of MCP-1 and IL-8 in endothelial cells is upregulated upon PPAR $\alpha$ activation (93). In addition, PPAR $\alpha$ ligands exert ROS-generating effects in unactivated macrophages (94). By contrast, fibrates increase $\mathrm{Cu}-\mathrm{Zn}$ superoxide dismutase and decrease NADPH oxidase in endothelial cells, potentially decreasing LDL oxidation (95). This suggests that PPAR $\alpha$ actions are distinct from inflammatory status, as demonstrated by MCP-1 upregulation in early atherosclerotic lesions and MCP-1 downregulation in late lesions during PPAR $\alpha$ activation (90). Antiatherogenic effects can be attributed to PPAR $\alpha$-dependent repression of CRP-induced MCP-1 expression (96), inhibition of the expression of ET-1 (14), inhibition of IL-1-induced IL-6 release (15), and inhibition of LPS-induced VCAM-1 expression $(16,97,98)$. Similarly, high IFN- $\gamma$ serum levels are observed in atherosclerotic patients, and IFN- $\gamma$ release by activated Tlymphocytes is blunted by PPAR $\alpha$ activators (99).

Furthermore, PPAR $\alpha$ has a critical role in controlling the cholesterol cycle in macrophages. The expression of ABCA1 is stimulated by PPAR $\alpha$ in foam cells in a liver $\mathrm{X}$ receptor-dependent manner, promoting apoA-I-mediated cholesterol efflux (62). The expression of Niemann-Pick type C proteins 1 and 2, transporters of cholesterol from lysosomes to the plasma membrane, is also regulated by PPAR $\alpha$, which promotes cholesterol availability for efflux (100). SR-BI, which plays a role in both the uptake of HDL-CE by the liver and cholesterol efflux from macrophages, is upregulated by PPAR $\alpha$ ligands in macrophages
(61), favoring cholesterol removal. Another parameter controlling macrophage cholesterol uptake is the availability and activity of released LPL. PPAR $\alpha$ reduces LPL secretion and decreases macrophage uptake of glycated LDL (101). FAO is also induced in macrophages, as in liver. CPT-1 expression is upregulated by PPAR $\alpha$ ligands, decreasing the FA pool available for cholesterol esterification (102).

Some of the later steps of atherosclerosis are also regulated by PPAR $\alpha$. Activation of SMC proliferation is a key event in atherosclerosis development and its complications. Upon vascular injury, SMCs migrate from the media into the neointimal layer of the vascular wall, where they proliferate and synthesize proteoglycans, leading to intimal hyperplasia. SMC proliferation is also one of the primary mechanisms underlying restenosis, an occlusive complication of corrective angioplasty procedures. PPAR $\alpha$ inhibits SMC proliferation by blocking G1/S cell cycle transition, through the induction of the cyclin-dependent kinase inhibitor $\mathrm{p} 16$. This results in SMC growth inhibition and reduced neointima formation in a mouse model of carotid artery injury (103). The migration of SMCs requires the degradation of the extracellular matrix by MMPs. Among them, MMP-9 contributes significantly to SMC migration, and its expression is reduced by PPAR $\alpha$ (104). Furthermore, by inhibiting the expression of tissue factor, a major procoagulant, PPAR $\alpha$ may block atherothrombosis $(105,106)$.

\section{PPAR $\alpha$ modulates hepatic inflammation}

Fibrates decrease the level of CRP, a major acute-phase protein stimulated by IL-1 and IL- 6 and a risk factor for cardiovascular disease. Synthesized in the liver, PPAR $\alpha$ ligands suppress CRP expression through an indirect transcriptional mechanism (19). The expression of fibrinogen- $\alpha$ and $-\beta$ and of serum amyloid A is repressed in a similar fashion (107). Thus PPAR $\alpha$ acts as an antiatherogenic factor by modulating local and systemic inflammatory responses, as well as lipid homeostasis in cell types that constitute the atherosclerotic plaque.

\section{Animal models of PPAR $\alpha$ action in atherosclerosis}

Despite a wealth of evidence documenting antiatherogenic properties of PPAR $\alpha$ ligands in vitro, mouse models have yielded contradictory results, which are furthermore difficult to extrapolate to human disease. First, mice are notoriously resistant to atherosclerosis, and only an aggressive diet rich in fat, cholesterol, and cholate and/or genetic manipulation, such as knockout of apoE or LDL receptor genes or knock-in of human apoE2, yields models that mimic some features of human dyslipidemia and atherosclerosis. Here again, the literature points to inconsistencies between effects of PPAR $\alpha$ agonists and the phenotype of PPAR $\alpha$-deficient mice. In the apoE $E^{-/-}$background, PPAR $\alpha$ deficiency was shown to protect mice from atherosclerosis, hinting at a proatherogenic role of PPAR $\alpha$ (74). However, $a p o E^{-/-}$mice fed a Western diet developed atherosclerotic lesions that regressed moderately upon fenofibrate treatment, an effect accentuated in the apo $E^{-/-}$strain expressing a human apoA-I transgene (89). In LDL receptor-deficient mice, another model of hypercholesterolemia, GW7647, a highly active PPAR $\alpha$ agonist, strongly decreases lesion formation (90). Similarly, in the apoE2 knock-in mouse, a model of mixed dyslipidemia, fenofibrates also lower lesion size (92). While these few examples highlight the inequities of knockout studies and agonist treatment, they also allude 
to the distinct pharmacokinetic properties of PPAR $\alpha$ ligands. Fibrates are known to act preferentially in the liver and are lowaffinity ligands for PPAR $\alpha$, whereas high-affinity ligands such as Wy14,643 and GW7647 are suspected of acting more efficiently in peripheral tissues. These discrepancies may also arise from differences in mouse and human lipid metabolism. In mice, HDL is the main transporter of cholesterol, whereas LDL is the principal carrier in humans. In addition, interspecies variation of the mode of regulation of metabolic genes may be considerable, as mentioned for the apoA-I gene. It is also worth noting that PPAR $\alpha$ has species-specific functional (39) and ligand-binding properties (108-110), so caution must be used in extrapolating data from the murine to the human situation.

Animal models are also used to identify PPAR $\alpha$-regulated pathologies other than atherosclerosis. For example, pretreatment of rats or mice with PPAR $\alpha$ agonists protects the heart from reperfusion injury induced by coronary occlusion (111, 112). Cerebral ischemia is also a major cause of stroke, and preventive treatment by fibrates reduces the susceptibility of mice to stroke and decreases cerebral infarct size $(113,114)$. These studies point to a potential preventive application of PPAR $\alpha$ ligands in such pathologies and, interestingly, expand the biological roles of PPAR $\alpha$ to other organs.

\section{Clinical consequences of PPAR $\alpha$ activation}

The actions of fibrates in humans have been tested in several clinical studies. Treatment with fibrates, such as fenofibrate, improves endothelial dysfunction in patients with type 2 diabetes (115). Evidence that PPAR $\alpha$ signaling is critical in the progression of atherosclerotic lesion formation in humans is provided by coronary angiography in both nondiabetic and diabetic patients. A decreased atherosclerosis progression was observed with gemfibrozil in the Lopid Coronary Angiography Trial, with bezafibrate in the Bezafibrate Coronary Atherosclerosis Intervention Trial, and with fenofibrate in the Diabetes Atherosclerosis Intervention Study (116-118). More importantly, the influence of fibrate treatment on cardiovascular morbidity and mortality was studied in primary (Helsinki Heart Study; FIELD) and secondary (Bezafibrate Infarction Prevention; Veterans Affairs High-Density Lipoprotein Cholesterol Intervention Trial; FIELD) prevention studies (88, 119-121). In the Helsinki Heart Study, cardiovascular disease risk reduction upon gemfibrozil treatment was most pronounced in overweight patients with metabolic syndrome or diabetes and atherogenic dyslipidemia $(122,123)$. In the Bezafibrate Infarction Prevention trial, reduction in coronary events with bezafibrate was observed only in patients with serum TG concentrations greater than $200 \mathrm{mg} / \mathrm{dl}$ (119), whereas the Veterans Affairs High-Density Lipoprotein Cholesterol Intervention Trial showed the most significant benefits of gemfibrozil in diabetics or in nondiabetics with high insulin levels (124). Altogether, these observations indicate that fibrates are particularly useful to treat the cardiovascular risk in insulin-resistant prediabetic individuals, and in diabetic patients with dyslipidemia. The most recent results come from the FIELD study (88), a combined primary and secondary prevention study testing the effects of fenofibrate on coronary heart disease in 9,795 type 2 diabetes patients who had no indication for lipid-lowering therapy. The primary endpoints, death from coronary heart disease or nonfatal myocardial infarction, were decreased, although not significantly, by $11 \%$ (mean follow-up, 5 years). However, during the course of the study, there was a gradual increase in statin use, which was greater in the placebo group than in the fenofibrate group. Since statins can decrease cardiovascular risk in type 2 diabetic patients (125), the actual benefit of fenofibrate thus may be underestimated because of the higher use of statins in the placebo arm. After correction for the statin effect, it was estimated that fenofibrate treatment resulted in a $19 \%$ reduction of relative risk of the primary endpoints. The benefits of fenofibrate were mainly due to reductions in nonfatal myocardial infarction and coronary revascularization. Moreover, fenofibrate treatment reduced microvascular complications (such as progression to microalbuminuria and intervention for retinopathy); this was not explained by changes in blood glucose control. Finally, the FIELD trial does not suggest that there are safety issues associated with fenofibrate-statin combination therapy. The major unanswered question is whether fenofibrate treatment confers additional benefit when given on top of a statin. This issue will be addressed in the ongoing Action to Control Cardiovascular Risk in Diabetes (ACCORD) study, the results of which are expected in early 2010 (S21).

\section{Conclusion}

The use of the PPAR $\alpha$ agonists, fibrates, as hypolipidemic agents for several decades has demonstrated their safety and efficacy for lipid lowering, an important parameter in the prevention of cardiovascular diseases. Moreover, increasing evidence attributing antiinflammatory activities to PPAR $\alpha$ emerges, documented largely in in vitro and animal studies. Prediabetic metabolic syndrome patients with atherogenic dyslipidemia (inflammation, low HDL, high TG, and sdLDL) are highly susceptible to cardiovascular morbidity and respond extremely well to fibrate treatment. In type 2 diabetics, fibrate treatment was recently demonstrated to reduce nonfatal myocardial infarction and coronary revascularization, suggesting beneficial effects of these drugs in these patients. Whether activation of PPAR $\alpha$ has detrimental effects in the hearts of diabetic patients, as observed after PPAR $\alpha$ overexpression in mouse cardiomyocytes, is unclear. However, at present, there is no indication that fibrate treatment would increase chronic heart insufficiency in humans; this points to possibly distinct responses of humans versus mice to chronic stimulation of cardiac metabolism by PPAR $\alpha$ activators. This view is strengthened by the fact that PPAR $\alpha$ activators exert species-specific activities and may induce peroxisome proliferation in mouse hearts, which could increase oxidative stress. Additionally, these species-specific responses are illustrated by the observation that fibrate treatment does not perturb glucose homeostasis in humans, although a negative effect could have been predicted from mouse data showing that PPAR $\alpha$ overexpression in skeletal muscle provokes insulin resistance. PPAR $\alpha$ activators appear to be particularly indicated to treat dyslipidemia of the metabolic syndrome and type 2 diabetes, although adverse effects on cardiac and skeletal muscle should be monitored in the development of novel, more potent PPAR $\alpha$ activators. Promising future developments undoubtedly lie in the field of selective PPAR $\alpha$ modulators (SPPARMs).

Note: References S1-S21 are available online with this article; doi:10.1172/JCI27989DS1.

Address correspondence to: Bart Staels, U.R. 545 INSERM-Institut Pasteur de Lille, 1 rue du Professeur Calmette, BP245, 59019 Lille, France. Phone: 33-320-87-73-88; Fax: 33-320-87-71-98; E-mail: bart.staels@pasteur-lille.fr. 
1. Issemann, I., and Green, S. 1990. Activation of a member of the steroid hormone receptor superfamily by peroxisome proliferators. Nature. 347:645-650

2. Forman, B.M., Chen, J., and Evans, R.M. 1997. Hypolipidemic drugs, polyunsaturated fatty acids and eicosanoids are ligands for peroxisome proliferator-activated receptors $\alpha$ and $\delta$. Proc. Natl. Acad. Sci.U.S. A. 94:4312-4317.

3. Lehmann, J.M., et al. 1995. An antidiabetic thiazolidinedione is a high affinity ligand for peroxisome proliferator-activated receptor $\gamma(\operatorname{PPAR} \gamma)$. J. Biol. Chem. 270:12953-12956.

4. Fu, J., et al. 2003. Oleylethanolamide regulates feeding and body weight through activation of the nuclear receptor PPAR $\alpha$. Nature. 425:90-93.

5. Devchand, P.R., et al. 1996. The PPAR $\alpha$-leukotriene B-4 pathway to inflammation control. Nature. 384:39-43.

6. Sumanasekera, W.K., et al. 2003. Heat shock protein-90 (hsp90) acts as a repressor of peroxisome proliferator-activated receptor $\alpha$ and PPAR $\gamma$ activity Biochemistry. 42:10726-10735.

7. Hi, R., Osada, S., Yumoto, N., and Osumi, T. 1999 Characterization of the amino-terminal activation domain of peroxisome proliferator-activated receptor $\alpha$. Importance of $\alpha$-helical structure in the transactivating function. J. Biol. Chem. 274:35152-35158.

8. Wan, Y.J.Y., et al. 2000. Peroxisome proliferatoractivated receptor $\alpha$-mediated pathways are altered in hepatocyte-specific retinoid X receptor $\alpha$-deficient mice. J. Biol. Chem. 275:28285-28290.

9. Ijpenberg, A., et al. 2004. In vivo activation of PPAR target genes by RXR homodimers. EMBO J. 23:2083-2091

10. Cronet, P., et al. 2001. Structure of the PPAR $\alpha$ and PPAR $\gamma$ ligand binding domain in complex with AZ 242 ; ligand selectivity and agonist activation in the PPAR family. Structure. 9:699-706.

11. Xu, H.E., et al. 2002. Structural basis for antagonist-mediated recruitment of nuclear co-repressors by PPAR $\alpha$. Nature. 415:813-817.

12. Duez, H., et al. 2005. Regulation of human ApoA-I by gemfibrozil and fenofibrate through selective peroxisome proliferator-activated receptor $\alpha$ modulation. Arterioscler. Thromb. Vasc. Biol. 25:585-591.

13. Qi, C., et al. 1999. Mouse steroid receptor coactivator- 1 is not essential for peroxisome proliferatoractivated receptor $\alpha$-regulated gene expression. Proc. Natl. Acad. Sci. U. S. A. 96:1585-1590.

14. Delerive, P., et al. 1999. Peroxisome proliferatoractivated receptor activators inhibit thrombininduced endothelin-1 production in human vascular endothelial cells by inhibiting the activator protein-1 signaling pathway. Circ. Res. 85:394-402.

15. Staels, B., et al. 1998. Activation of human aortic smooth-muscle cells is inhibited by PPAR $\alpha$ but not by PPAR $\gamma$ activators. Nature. 393:790-793

16. Marx, N., Sukhova, G.K., Collins, T., Libby, P., and Plutzky, J. 1999. PPAR $\alpha$ activators inhibit cytokine-induced vascular cell adhesion molecule-1 expression in human endothelial cells. Circulation. 99:3125-3131.

17. Delerive, P., Gervois, P., Fruchart, J.C., and Staels, B. 2000. Induction of Ikappa-B $\alpha$ expression as a mechanism contributing to the anti-inflammatory activities of peroxisome proliferatoractivated receptor- $\alpha$ activators. J. Biol. Chem. 275:36703-36707

18. Delerive, P., et al. 2002. DNA binding-independent induction of IkappaB $\alpha$ gene transcription by PPARa. Mol. Endocrinol. 16:1029-1039.

19. Kleemann, R., et al. 2003. Fibrates down-regulate IL-1-stimulated C-reactive protein gene expression in hepatocytes by reducing nuclear $\mathrm{p} 50$ NFkappa B-C/EBP- $\beta$ complex formation. Blood. 101:545-551.
20. Gervois, P., et al. 2001. Negative regulation of human fibrinogen gene expression by peroxisome proliferator-activated receptor $\alpha$ agonists via inhibition of CCAAT Box/Enhancer-binding protein $\beta$. J. Biol. Chem. 276:33471-33477.

21. Gervois, P., et al. 2004. Global suppression of IL-6induced acute phase response gene expression after chronic in vivo treatment with the peroxisome proliferator-activated receptor- $\alpha$ activator fenofibrate. J. Biol. Chem. 279:16154-16160.

22. Blanquart, C., et al. 2004. The protein kinase C signaling pathway regulates a molecular switch between transactivation and transrepression activity of the peroxisome proliferator-activated receptor $\alpha$ Mol. Endocrinol. 18:1906-1918.

23. Lemberger, T., et al. 1996. Expression of the peroxisome proliferator-activated receptor $\alpha$ gene is stimulated by stress and follows a diurnal rhythm. J. Biol. Chem. 271:1764-1769.

24. Steineger, H.H., et al. 1994. Dexamethasone and insulin demonstrate marked and opposite regulation of the steady-state mRNA level of the peroxisomal proliferator-activated receptor (PPAR) in hepatic cells. Hormonal modulation of fatty-acid-induced transcription. Eur. J. Biochem. 225:967-974

25. Lemberger, T., et al. 1994. Regulation of the peroxisome proliferator-activated receptor $\alpha$ gene by glucocorticoids. J. Biol. Chem. 269:24527-24530.

26. Oishi, K., Shirai, H., and Ishida, N. 2005. CLOCK is involved in the circadian transactivation of peroxisomeproliferator-activated receptor $\alpha(\operatorname{PPAR} \alpha)$ in mice. Biochem. J. 386:575-581.

27. Leone, T.C., Weinheimer, C.J., and Kelly, D.P. 1999. A critical role for the peroxisome proliferator-activated receptor $\alpha(\operatorname{PPAR} \alpha)$ in the cellular fasting response: the PPAR $\alpha$-null mouse as a model of fatty acid oxidation disorders. Proc. Natl. Acad. Sci. U. S. A. 96:7473-7478.

28. Kersten, S., et al. 1999. Peroxisome proliferator-activated receptor $\alpha$ mediates the adaptive response to fasting. J. Clin. Invest. 103:1489-1498.

29. Zhou, Y.T., et al. 1998. Role of peroxisome proliferator-activated receptor $\alpha$ in disease of pancreatic $\beta$ cells. Proc. Natl. Acad. Sci. U. S. A. 95:8898-8903.

30. Jalouli, M., et al. 2003. Sex difference in hepatic peroxisome proliferator-activated receptor $\alpha$ expression: influence of pituitary and gonadal hormones. Endocrinology. 144:101-109.

31. Blanquart, C., Barbier, O., Fruchart, J.C., Staels, B., and Glineur, C. 2002. Peroxisome proliferator-activated receptor $\alpha$ (PPAR $\alpha)$ turnover by the ubiquitin-proteasome system controls the ligandinduced expression level of its target genes. J. Biol. Chem. 277:37254-37259.

32. Pineda Torra, I., Jamshidi, Y., Flavell, D.M., Fruchart, J.C., and Staels, B. 2002. Characterization of the human PPAR $\alpha$ promoter: identification of a functional nuclear receptor response element. Mol. Endocrinol. 16:1013-1028.

33. Roduit, R., et al. 2000. Glucose down-regulates the expression of the peroxisome proliferator-activated receptor- $\alpha$ gene in the pancreatic $\beta$-cell. J. Biol. Chem. 275:35799-35806.

34. Barger, P.M., Browning, A.C., Garner, A.N., and Kelly, D.P. 2001. p38 mitogen-activated protein kinase activates peroxisome proliferator-activated receptor $\alpha$. A potential role in the cardiac metabolic stress response. J. Biol. Chem. 276:44495-44501.

35. Kliewer, S.A., et al. 1997. Fatty acids and eicosanoids regulate gene expression through direct interactions with peroxisome proliferator-activated receptors $\alpha$ and $\gamma$. Proc. Natl. Acad. Sci. U. S. A. 94:4318-4323.

36. Hostetler, H.A., Petrescu, A.D., Kier, A.B., and Schroeder, F. 2005. Peroxisome proliferator-activated receptor $\alpha$ interacts with high affinity and is conformationally responsive to endogenous ligands. J. Biol. Chem. 280:18667-18682.

37. Xu, H.E., et al. 1999. Molecular recognition of fatty acids by peroxisome proliferator-activated receptors. Mol. Cell. 3:397-403.

38. Chakravarthy, M.V., et al. 2005. "New" hepatic fat activates PPAR $\alpha$ to maintain glucose, lipid, and cholesterol homeostasis. Cell Metab. 1:309-322.

39. Cheung, C., et al. 2004. Diminished hepatocellular proliferation in mice humanized for the nuclear receptor peroxisome proliferator-activated receptor $\alpha$. Cancer Res. 64:3849-3854

40. Aoyama, T., et al. 1998. Altered constitutive expression of fatty acid-metabolizing enzymes in mice lacking the peroxisome proliferator-activated receptor $\alpha(\mathrm{PPAR} \alpha)$. J. Biol. Chem. 273:5678-5684.

41. Lee, S.S., et al. 1995. Targeted disruption of the $\alpha$ isoform of the peroxisome proliferator-activated receptor gene in mice results in abolishment of the pleiotropic effects of peroxisome proliferators. $\mathrm{Mol}$. Cell. Biol. 15:3012-3022.

42. Peters, J.M., et al. 1997. Alterations in lipoprotein metabolism in peroxisome proliferatoractivated receptor $\alpha$-deficient mice. J. Biol. Chem. 272:27307-27312.

43. Djouadi, F., et al. 1998. A gender-related defect in lipid metabolism and glucose homeostasis in peroxisome proliferator-activated receptor $\alpha$-deficient mice. J. Clin. Invest. 102:1083-1091.

44. Brandt, J.M., Djouadi, F., and Kelly, D.P. 1998. Fatty acids activate transcription of the muscle carnitine palmitoyltransferase I gene in cardiac myocytes via the peroxisome proliferator-activated receptor $\alpha$. J. Biol. Chem. 273:23786-23792.

45. Rodriguez, J.C., Gil-Gomez, G., Hegardt, F.G., and Haro, D. 1994. Peroxisome proliferator-activated receptor mediates induction of the mitochondrial 3-hydroxy-3-methylglutaryl-CoA synthase gene by fatty acids. J. Biol. Chem. 269:18767-18772.

46. Martin, G., Schoonjans, K., Lefebvre, A.M., Staels, B., and Auwerx, J. 1997. Coordinate regulation of the expression of the fatty acid transport protein and acyl-CoA synthetase genes by PPAR $\alpha$ and PPAR $\gamma$ activators. J. Biol. Chem. 272:28210-28217.

47. Palmer, C.N., Hsu, M.H., Griffin, K.J., Raucy, J.L., and Johnson, E.F. 1998. Peroxisome proliferator activated receptor- $\alpha$ expression in human liver. Mol. Pharmacol. 53:14-22.

48. Gervois, P., et al. 1999. A truncated human peroxisome proliferator-activated receptor $\alpha$ splice variant with dominant negative activity. Mol. Endocrinol. 13:1535-1549.

49. Motojima, K., Passilly, P., Peters, J.M., Gonzalez, F.J., and Latruffe, N. 1998. Expression of putative fatty acid transporter genes are regulated by peroxisome proliferator-activated receptor $\alpha$ and $\gamma$ activators in a tissue- and inducer-specific manner. J. Biol. Chem. 273:16710-16714.

50. Lee, S.S., et al. 1995. Targeted disruption of the $\alpha$ isoform of the peroxisome proliferator-activated receptor gene in mice results in abolishment of the pleiotropic effects of peroxisome proliferators. $\mathrm{Mol}$. Cell. Biol. 15:3012-3022.

51. Finck, B.N., et al. 2002. The cardiac phenotype induced by PPAR $\alpha$ overexpression mimics that caused by diabetes mellitus. J. Clin. Invest. 109:121-130. doi:10.1172/JCI200214080.

52. Gilde, A.J., et al. 2003. Peroxisome proliferatoractivated receptor (PPAR) $\alpha$ and PPAR $\beta / \delta$, but not PPAR $\gamma$, modulate the expression of genes involved in cardiac lipid metabolism. Circ. Res. 92:518-524.

53. Park, S.Y., et al. 2005. Cardiac-specific overexpression of peroxisome proliferator-activated receptor- $\alpha$ causes insulin resistance in heart and liver. Diabetes. 54:2514-2524.

54. Barger, P.M., Brandt, J.M., Leone, T.C., Weinheimer, C.J., and Kelly, D.P. 2000. Deactivation of peroxisome proliferator-activated receptor- $\alpha$ during cardiac hypertrophic growth. J. Clin. Invest. 
105:1723-1730.

55. Finck, B.N., et al. 2003. A critical role for PPAR $\alpha$ mediated lipotoxicity in the pathogenesis of diabetic cardiomyopathy: modulation by dietary fat content. Proc. Natl. Acad. Sci. U. S. A. 100:1226-1231.

56. Vikramadithyan, R.K., et al. 2005. Peroxisome proliferator-activated receptor agonists modulate heart function in transgenic mice with lipotoxic cardiomyopathy. J. Pharmacol. Exp. Ther. 313:586-593.

57. Schoonjans, K., et al. 1996. PPAR $\alpha$ and PPAR $\gamma$ activators direct a distinct tissue-specific transcriptional response via a PPRE in the lipoprotein lipase gene. EMBO J. 15:5336-5348.

58. Staels, B., et al. 1995. Fibrates downregulate apolipoprotein C-III expression independent of induction of peroxisomal acyl coenzyme A oxidase. A potential mechanism for the hypolipidemic action of fibrates. J. Clin. Invest. 95:705-712.

59. Vu-Dac, N., et al. 2003. Apolipoprotein A5, a crucial determinant of plasma TG levels, is highly responsive to peroxisome proliferator-activated receptor $\alpha$ activators. J. Biol. Chem. 278:17982-17985.

60. Eriksson, M., Carlson, L.A., Miettinen, T.A., and Angelin, B. 1999. Stimulation of fecal steroid excretion after infusion of recombinant proapolipoprotein A-I. Potential reverse cholesterol transport in humans. Circulation. 100:594-598.

61. Chinetti, G., et al. 2000. CLA-1/SR-BI is expressed in atherosclerotic lesion macrophages and regulated by activators of peroxisome proliferator-activated receptors. Circulation. 101:2411-2417.

62. Chinetti, G., et al. 2001. PPAR- $\alpha$ and PPAR- $\gamma$ activators induce cholesterol removal from human macrophage foam cells through stimulation of the ABCA1 pathway. Nat. Med. 7:53-58.

63. Hennuyer, N., et al. 1999. Beneficial effects of fibrates on apolipoprotein A-I metabolism occur independently of any peroxisome proliferative response. Circulation. 99:2445-2451.

64. Vu-Dac, N., et al. 1994. Negative regulation of the human apolipoprotein A-I promoter by fibrates can be attenuated by the interaction of the peroxisome proliferator-activated receptor with its response element. J. Biol. Chem. 269:31012-31018.

65. Watts, G.F., et al. 2003. Differential regulation of lipoprotein kinetics by atorvastatin and fenofibrate in subjects with the metabolic syndrome. Diabetes. 52:803-811.

66. Vu-Dac, N., et al. 1995. Fibrates increase human apolipoprotein A-II expression through activation of the peroxisome proliferator-activated receptor. J. Clin. Invest. 96:741-750.

67. Vu-Dac, N., et al. 1998. The nuclear receptors peroxisome proliferator-activated receptor $\alpha$ and Rev-erb $\alpha$ mediate the species-specific regulation of apolipoprotein A-I expression by fibrates. J. Biol. Chem. 273:25713-25720.

68. Bandsma, R.H.J., et al. 2004. Hepatic de-novo synthesis of glucose 6-phosphate is not affected in peroxisome proliferator-activated receptor $\alpha$-deficient mice but is preferentially directed toward hepatic glycogen stores after a short term fast. J. Biol. Chem. 279:8930-8937.

69. Xu, J., et al. 2002. Peroxisome proliferator-activated receptor $\alpha(P P A R \alpha)$ influences substrate utilization for hepatic glucose production. J. Biol. Chem. 277:50237-50244.

70. Patsouris, D., et al. 2004. PPAR $\alpha$ governs glycerol metabolism. J. Clin. Invest. 114:94-103. doi:10.1172/ JCI200420468.

71. Koo, S.H., et al. 2004. PGC-1 promotes insulin resistance in liver through PPAR- $\alpha$-dependent induction of TRB-3. Nat. Med. 10:530-534.

72. Bernal-Mizrachi, C., et al. 2003. Dexamethasone induction of hypertension and diabetes is PPAR-o dependent in LDL receptor-null mice. Nat. Med. 9:1069-1075.
73. Guerre-Millo, M., et al. 2001. PPAR $\alpha$-null mice are protected from high-fat diet-induced insulin resistance. Diabetes. 50:2809-2814.

74. Tordjman, K., et al. 2001. PPAR $\alpha$ deficiency reduces insulin resistance and atherosclerosis in ApoE-null mice. J. Clin. Invest. 107:1025-1034.

75. Gremlich, S., et al. 2005. Pancreatic islet adaptation to fasting is dependent on peroxisome proliferatoractivated receptor $\alpha$ transcriptional up-regulation of fatty acid oxidation. Endocrinology. 146:375-382.

76. Guerre-Millo, M., et al. 2000. Peroxisome proliferator-activated receptor $\alpha$ activators improve insulin sensitivity and reduce adiposity. J. Biol. Chem. 275:16638-16642.

77. Chou, C.J., et al. 2002. WY14,643, a peroxisome proliferator-activated receptor $\alpha$ (PPAR $\alpha)$ agonist, improves hepatic and muscle steatosis and reverses insulin resistance in lipoatrophic A-ZIP/F-1 mice. J. Biol. Chem. 277:24484-24489.

78. Kim, H., et al. 2003. Peroxisome proliferator-activated receptor- $\alpha$ agonist treatment in a transgenic model of type 2 diabetes reverses the lipotoxic state and improves glucose homeostasis. Diabetes. 52:1770-1778

79. Schafer, S.A., Hansen, B.C., Volkl, A., Fahimi, H.D., and Pill, J. 2004. Biochemical and morphological effects of K-111, a peroxisome proliferator-activated receptor (PPAR)- $\alpha$ activator, in non-human primates. Biochem. Pharmacol. 68:239-251.

80. Ye, J.M., et al. 2001. Peroxisome proliferator-activated receptor (PPAR)- $\alpha$ activation lowers muscle lipids and improves insulin sensitivity in high fat-fed rats: comparison with PPAR- $\gamma$ activation. Diabetes. 50:411-417.

81. Ravnskjaer, K., et al. 2005. Peroxisome proliferatoractivated receptor $\alpha$ (PPAR $\alpha)$ potentiates, whereas PPAR $\gamma$ attenuates, glucose-stimulated insulin secretion in pancreatic $\beta$-cells. Endocrinology. 146:3266-3276.

82. Muoio, D.M., et al. 2002. Fatty acid homeostasis and induction of lipid regulatory genes in skeletal muscles of peroxisome proliferator-activated receptor (PPAR) $\alpha$ knock-out mice. Evidence for compensatory regulation by PPAR $\delta$. J. Biol. Chem. 277:26089-26097.

83. Finck, B.N., et al. 2005. A potential link between muscle peroxisome proliferator-activated receptor- $\alpha$ signaling and obesity-related diabetes. Cell Metab. 1:133-144.

84. Steiner, G. 1991. Altering TG concentrations changes insulin-glucose relationships in hypertriglyceridemic patients. Double-blind study with gemfibrozil with implications for atherosclerosis. Diabetes Care. 14:1077-1081.

85. Ferrari, C., et al. 1977. Effects of short-term clofibrate administration on glucose tolerance and insulin secretion in patients with chemical diabetes or hypertriglyceridemia. Metabolism. 26:129-139.

86. Murakami, K., et al. 1984. Clofibrate enhances the affinity of insulin receptors in non-insulin dependent diabetes mellitus. Br. J. Clin. Pharmacol. 17:89-91.

87. Kobayashi, M., et al. 1988. Improvement of glucose tolerance in NIDDM by clofibrate. Randomized double-blind study. Diabetes Care. 11:495-499.

88. Keech, A., et al. 2005. Effects of long-term fenofibrate therapy on cardiovascular events in 9795 people with type 2 diabetes mellitus (the FIELD study): randomised controlled trial. Lancet. 366:1849-1861.

89. Duez, H., et al. 2002. Reduction of atherosclerosis by the peroxisome proliferator-activated receptor $\alpha$ agonist fenofibrate in mice. J. Biol. Chem 277:48051-48057.

90. Li, A.C., et al. 2004. Differential inhibition of macrophage foam-cell formation and atherosclerosis in mice by PPAR $\alpha, \beta / \delta$, and $\gamma$. J. Clin. Invest. 114:1564-1576. doi:10.1172/JCI200418730.
91. Fu, T., Mukhopadhyay, D., Davidson, N.O., and Borensztajn, J. 2004. The peroxisome proliferator-activated receptor $\alpha$ (PPAR $\alpha$ ) agonist ciprofibrate inhibits apolipoprotein B mRNA editing in low density lipoprotein receptor-deficient mice: effects on plasma lipoproteins and the development of atherosclerotic lesions. J. Biol. Chem. 279:28662-28669.

92. Hennuyer, N., et al. 2005. PPAR $\alpha$, but not PPAR $\gamma$, activators decrease macrophage-laden atherosclerotic lesions in a nondiabetic mouse model of mixed dyslipidemia. Arterioscler. Thromb. Vasc. Biol. 25:1897-1902.

93. Lee, H., et al. 2000. Role for peroxisome proliferator-activated receptor $\alpha$ in oxidized phospholipidinduced synthesis of monocyte chemotactic protein- 1 and interleukin- 8 by endothelial cells. Circ. Res. 87:516-521.

94. Teissier, E., et al. 2004. Peroxisome proliferatoractivated receptor $\alpha$ induces NADPH oxidase activity in macrophages, leading to the generation of LDL with PPAR- $\alpha$ activation properties. Circ. Res. 95:1174-1182.

95. Inoue, I., et al. 2001. The ligands/activators for peroxisome proliferator-activated receptor $\alpha$ (PPAR $\alpha)$ and PPAR $\gamma$ increase $\mathrm{Cu}^{2+}, \mathrm{Zn}^{2+}$-superoxide dismutase and decrease $\mathrm{p} 22$ phox message expressions in primary endothelial cells. Metabolism. 50:3-11.

96. Pasceri, V., Cheng, J.S., Willerson, J.T., and Yeh, E.T. 2001. Modulation of C-reactive protein-mediated monocyte chemoattractant protein-1 induction in human endothelial cells by anti-atherosclerosis drugs. Circulation. 103:2531-2534.

97. Jackson, S.M., et al. 1999. Peroxisome proliferator-activated receptor activators target human endothelial cells to inhibit leukocyte-endothelial cell interaction. Arterioscler. Thromb. Vasc. Biol. 19:2094-2104.

98. Rival, Y., et al. 2002. PPAR $\alpha$ and PPAR $\delta$ activators inhibit cytokine-induced nuclear translocation of NF-kappaB and expression of VCAM-1 in EAhy926 endothelial cells. Eur. J. Pharmacol. 435:143-151.

99. Marx, N., et al. 2002. PPAR activators as antiinflammatory mediators in human $\mathrm{T}$ lymphocytes: implications for atherosclerosis and transplantationassociated arteriosclerosis. Circ. Res. 90:703-710.

100.Chinetti-Gbaguidi, G., et al. 2005. Peroxisome proliferator-activated receptor $\alpha$ controls cellular cholesterol trafficking in macrophages. J. Lipid Res. 46:2717-2725.

101. Gbaguidi, F.G., et al. 2002. Peroxisome proliferatoractivated receptor (PPAR) agonists decrease lipoprotein lipase secretion and glycated LDL uptake by human macrophages. FEBS Lett. 512:85-90.

102. Chinetti, G., Lestavel, S., Fruchart, J.C., Clavey, V., and Staels, B. 2003. Peroxisome proliferator-activated receptor $\alpha$ reduces cholesterol esterification in macrophages. Circ. Res. 92:212-217.

103.Gizard, F., et al. 2005. PPAR $\alpha$ inhibits vascular smooth muscle cell proliferation underlying intimal hyperplasia by inducing the tumor suppressor p16. J. Clin. Invest. 115:3228-3238. doi:10.1172/ JCI22756.

104.Shu, H., et al. 2000. Activation of PPAR $\alpha$ or $\gamma$ reduces secretion of matrix metalloproteinase 9 but not interleukin 8 from human monocytic THP-1 cells. Biochem. Biophys. Res. Commun. 267:345-349.

105.Marx, N., et al. 2001. PPAR $\alpha$ activators inhibit tissue factor expression and activity in human monocytes. Circulation. 103:213-219.

106.Neve, B.P., et al. 2001. PPAR $\alpha$ agonists inhibit tissue factor expression in human monocytes and macrophages. Circulation. 103:207-212.

107. Gervois, P., et al. 2001. Negative regulation of human fibrinogen gene expression by peroxisome proliferator-activated receptor $\alpha$ agonists via inhibition of CCAAT box/enhancer-binding protein $\beta$. J. Biol. Chem. 276:33471-33477. 
108. Nagasawa, M., et al. 2004. Pharmacological characterization of a human-specific peroxisome proliferator-activated receptor $\alpha(\operatorname{PPAR} \alpha)$ agonist in dogs. Biochem. Pharmacol. 67:2057-2069.

109. Mukherjee, R., Jow, L., Noonan, D., and McDonnell, D.P. 1994. Human and rat peroxisome proliferator activated receptors (PPARs) demonstrate similar tissue distribution but different responsiveness to PPAR activators. J. Steroid Biochem. Mol. Biol. 51:157-166.

110. Keller, H., Devchand, P.R., Perroud, M., and Wahli, W. 1997. PPAR $\alpha$ structure-function relationships derived from species-specific differences in responsiveness to hypolipidemic agents. Biol. Chem. 378:651-655.

111.Yue, T.-L., et al. 2003. Activation of peroxisome proliferator-activated receptor $\alpha$ protects the heart from ischemia/reperfusion injury. Circulation. 108:2393-2399.

112. Wayman, N.S., et al. 2002. Ligands of the peroxisome proliferator-activated receptors (PPAR $\gamma$ and PPAR $\alpha$ ) reduce myocardial infarct size. FASEB J. 16:1027-1040

113. Deplanque, D., et al. 2003. Peroxisome proliferator-activated receptor $\alpha$ activation as a mechanism of preventive neuroprotection induced by chronic fenofibrate treatment. J. Neurosci. 23:6264-6271.

114.Inoue, H., et al. 2003. Brain protection by resveratrol and fenofibrate against stroke requires peroxi- some proliferator-activated receptor $\alpha$ in mice. Neurosci. Lett. 352:203-206.

115.Playford, D.A., Watts, G.F., Best, J.D., and Burke, V. 2002. Effect of fenofibrate on brachial artery flowmediated dilatation in type 2 diabetes mellitus. Am. J. Cardiol. 90:1254-1257.

116.Frick, M.H., et al. 1997. Prevention of the angiographic progression of coronary and vein-graft atherosclerosis by gemfibrozil after coronary bypass surgery in men with low levels of HDL cholesterol. Lopid Coronary Angiography Trial (LOCAT) Study Group. Circulation. 96:2137-2143.

117. Ruotolo, G., et al. 1998. Treatment effects on serum lipoprotein lipids, apolipoproteins and low density lipoprotein particle size and relationships of lipoprotein variables to progression of coronary artery disease in the Bezafibrate Coronary Atherosclerosis Intervention Trial (BECAIT). J. Am. Coll. Cardiol. 32:1648-1656.

118. [Anonymous]. 2001. Effect of fenofibrate on progression of coronary-artery disease in type 2 diabetes: the Diabetes Atherosclerosis Intervention Study, a randomised study. Lancet. 357:905-910.

119. [Anonymous]. 2000. Secondary prevention by raising HDL cholesterol and reducing triglycerides in patients with coronary artery disease: the Bezafibrate Infarction Prevention (BIP) study. Circulation. 102:21-27.

120.Frick, M.H., et al. 1987. Helsinki Heart Study: pri- mary-prevention trial with gemfibrozil in middleaged men with dyslipidemia. Safety of treatment, changes in risk factors, and incidence of coronary heart disease. N. Engl. J. Med. 317:1237-1245.

121. Rubins, H.B., et al. 1999. Gemfibrozil for the secondary prevention of coronary heart disease in men with low levels of high-density lipoprotein cholesterol. Veterans Affairs High-Density Lipoprotein Cholesterol Intervention Trial Study Group. N. Engl. J. Med. 341:410-418.

122.Manninen, V., et al. 1992. Joint effects of serum TG and LDL cholesterol and HDL cholesterol concentrations on coronary heart disease risk in the Helsinki Heart Study. Implications for treatment. Circulation. 85:37-45.

123.Tenkanen, L., Manttari, M., and Manninen, V. 1995. Some coronary risk factors related to the insulin resistance syndrome and treatment with gemfibrozil. Experience from the Helsinki Heart Study. Circulation. 92:1779-1785.

124. Robins, S.J., et al. 2003. Insulin resistance and cardiovascular events with low HDL cholesterol: the Veterans Affairs HDL Intervention Trial (VA-HIT). Diabetes Care. 26:1513-1517.

125.Colhoun, H.M., et al. 2004. Primary prevention of cardiovascular disease with atorvastatin in type 2 diabetes in the Collaborative Atorvastatin Diabetes Study (CARDS): multicentre randomized placebocontrolled trial. Lancet. 364:685-696. 